\title{
MONOTONICITY OF POSITIVE SEMIDEFINITE HERMITIAN MATRICES ${ }^{1}$
}

\author{
RUSSELL MERRIS AND STEPHEN PIERCE
}

\begin{abstract}
Inequalities which compare elements of the convex cone of positive semidefinite hermitian matrices with products of roots of elements are proved. They yield inequalities for Schur functions (generalized matrix functions) which, when specialized to the determinant, give a result of R. Bellman and L. Mirsky.
\end{abstract}

Introduction. The following result was proved in [2] and [7]. (See also $\left[1\right.$, p. 63] and [4, p. 115].) Let $\theta_{1}, \cdots, \theta_{k}$ be positive numbers such that $\theta_{1}+\cdots+\theta_{k}=1$. Let $A_{1}, \cdots, A_{k}$ be positive definite hermitian $n$-square matrices. Then

$$
\operatorname{det}\left(\sum_{i=1}^{k} \theta_{i} A_{i}\right) \geqq \prod_{i=1}^{k}\left(\operatorname{det}\left(A_{i}\right)\right)^{\theta_{i}}
$$

with equality if and only if $A_{1}=\cdots=A_{k}$.

In this note, we observe that the above result generalizes in several directions (Theorems 2, 3, and 4). Theorem 1 provides the case of equality in several of our inequalities.

When $A$ is positive semidefinite (positive definite) hermitian, we write $A \geqq 0(A>0)$. If $A \geqq 0, B \geqq 0, A-B \geqq 0$, we write $A \geqq B$.

Results. Let $H$ be a subgroup of $S_{n}$, the symmetric group. Let $\chi$ be a character on $H$. Define $d(A)$, which depends on $H$ and $\chi$, by

$$
d(A)=\sum_{\sigma \in H} \chi(\sigma) \prod_{t=1}^{n} a_{t \sigma(t)}
$$

where $A=\left(a_{i j}\right)$. These functions were studied by Schur [8]. If $H=S_{n}$ and $\chi=$ sgn, then $d=\operatorname{det}$. If $H=S_{n}$ and $\chi \equiv 1, d=$ per (permanent).

We will frequently use the result in [6] that $A \geqq B$ implies $d(A) \geqq d(B)$.

TheOREM 1. Suppose $\chi \equiv 1$ or $d=$ det. If $A \geqq B$ and $d(A)=d(B) \neq 0$, then $A=B$.

We will prove Theorem 1 later.

Received by the editors September 21, 1970 and, in revised form, April 12, 1971. AMS 1970 subject classifications. Primary 15A57, 15A45; Secondary 15A15, 15 A69. Key words and phrases. Symmetric group, character, tensor power, Kronecker power.

${ }^{1}$ This work was done while the authors were National Academy of SciencesNational Research Council Postdoctoral Research Associates at the National Bureau of Standards, Washington, D.C. 20234.

(c) American Mathematical Society 1972 
THEOREM 2. Let $A_{i} \geqq 0, i=1, \cdots, k$, and suppose the $A_{i}$ are pairwise commuting. If $\theta_{1}, \cdots, \theta_{k}$ are positive numbers such that $\theta_{1}+\cdots+\theta_{k}=1$, then

$$
\sum_{i=1}^{k} \theta_{i} A_{i} \geqq \prod_{i=1}^{k} A_{i}^{\theta_{i}}
$$

with equality if and only if $A_{1}=\cdots=A_{k}$. Thus,

$$
d\left(\sum_{i=1}^{k} \theta_{i} A_{i}\right) \geqq d\left(\prod_{i=1}^{k} A_{i}^{\theta_{i}}\right) .
$$

If $\chi \equiv 1$ or $d=\operatorname{det}$, equality holds in (1) if and only if $A_{1}=\cdots=A_{k}$.

Proof. Since the $A_{i}$ 's commute, they can be simultaneously diagonalized. The result then follows from the arithmetic-geometric mean inequality and Theorem 1.

Theorem 3. Let $A_{1}, \cdots, A_{k}$ be as in Theorem 2. Then,

$$
d\left(\left(\sum_{i=1}^{k} \theta_{i} A_{i}\right)^{1 / n}\right) \geqq \prod_{i=1}^{k}\left(d\left(A_{i}^{1 / n}\right)\right)^{\theta_{i}}
$$

Proof. By the extended Minkowski inequality [3],

$$
d\left(\left(\sum_{i=1}^{k} \theta_{i} A_{i}\right)^{1 / n}\right) \geqq \sum_{i=1}^{k} \theta_{i} d\left(A_{i}^{1 / n}\right) .
$$

The arithmetic-geometric mean inequality now yields (2).

Without assuming commutativity, it is difficult to obtain much information on Schur functions other than the determinant. Generalizing Mirsky's method, we can prove

TheOREM 4. Let $A \geqq 0, B=C^{*} C>0$. Let $0<\theta<1$. Then

$$
\theta A+(1-\theta) B \geqq C^{*}\left(C^{*-1} A C^{-1}\right)^{\theta} C
$$

with equality if and only if $A=B$. Thus

$$
d(\theta A+(1-\theta) B) \geqq d\left(C^{*}\left(C^{*-1} A C^{-1}\right)^{\theta} C\right) .
$$

If $d=\operatorname{det}$ or $\chi \equiv 1$, equality holds in (3) if and only if $A=B$.

Proof. For $\lambda \geqq 0, \theta \lambda+1-\theta \geqq \lambda^{\theta}$ with equality if and only if $\lambda=1$. Thus, for any $H \geqq 0$,

$$
\theta H+(1-\theta) I \geqq H^{\theta}
$$

with equality if and only if $H=I$. Take $H=C^{*-1} A C^{-1}$.

The Bellman-Mirsky result follows from any of Theorems 2,3 , or 4, using the multiplicativity of the determinant. 
Proof OF TheOREM 1. Let $\lambda_{1} \geqq \cdots \geqq \lambda_{n} \geqq 0, \mu_{1} \geqq \cdots \geqq \mu_{n} \geqq 0$ be the eigenvalues of $A$ and $B$ respectively. A standard argument using the Courant-Fischer minimax theorem proves that $A \geqq B$ implies $\lambda_{i} \geqq \mu_{i}$, $i=1, \cdots, n$. Thus if $A \geqq B$ and $\operatorname{det}(A)=\operatorname{det}(B) \neq 0, \lambda_{i}=\mu_{i}, i=1, \cdots, n$. Hence trace $(A-B)=0$, and $A=B$.

If $\chi \equiv 1$, our proof requires the machinery of the associated transformation.

Let $V$ be the inner product space of column $n$-tuples. Let $E=\left\{e_{1}, \cdots, e_{n}\right\}$ be the standard basis, i.e., $e_{j}$ has a 1 in position $j$ and 0 elsewhere. Let $\otimes V$ be the $n$th tensor power of $V$. If $x_{1}, \cdots, x_{n} \in V$, write $x_{1} \otimes \cdots \otimes x_{n}$ as their tensor product. For $\sigma \in S_{n}$, let $P\left(\sigma^{-1}\right)$ be the linear operator defined by

Set

$$
P\left(\sigma^{-1}\right) x_{1} \otimes \cdots \otimes x_{n}=x_{\sigma(1)} \otimes \cdots \otimes x_{\sigma(n)}, \quad x_{i} \in V .
$$

$$
T(H, \chi)=\frac{1}{h} \sum_{\sigma \in H} \chi(\sigma) P(\sigma),
$$

where $h$ is the order of $H$. Write $W$ for the range of $T(H, \chi)$. Let

$$
x_{1} * \cdots * x_{n}=T(H, \chi) x_{1} \otimes \cdots \otimes x_{n} .
$$

Let $\otimes A$ denote the $n$th Kronecker power of $A$, i.e.,

$$
(\otimes A) x_{1} \otimes \cdots \otimes x_{n}=A x_{1} \otimes \cdots \otimes A x_{n} .
$$

The space $W$ is invariant under $\otimes A$ since $T(H, \chi)$ and $\otimes A$ commute. So, let $K(A)$ be the restriction of $\otimes A$ to $W$.

The inner product $(\cdot, \cdot)$ on $V$ induces an inner product on $\otimes V$ which satisfies

$$
\left(x_{1} \otimes \cdots \otimes x_{n}, y_{1} \otimes \cdots \otimes y_{n}\right)=\prod_{t=1}^{n}\left(x_{t}, y_{t}\right) .
$$

It has been proved, [5, p. 318], that there is a set $\Delta$ of integer sequences of length $n$ such that the set

$$
\left\{e_{\alpha}^{*}=e_{\alpha_{1}} * \cdots * e_{\alpha_{n}}: \alpha=\left(\alpha_{1}, \cdots, \alpha_{n}\right) \in \Delta\right\}
$$

forms an orthogonal basis of $W$. Moreover, we may assume $(1, \cdots, n) \in \Delta$.

For each $\alpha \in \Delta$, let $k_{\alpha}$ be the inverse of the norm of $e_{\alpha}^{*}$. Then

$$
E^{*}=\left\{k_{\alpha} e_{\alpha}^{*}: \alpha \in \Delta\right\}
$$

is an orthonormal basis of $W$. (When $\alpha=(1, \cdots, n), k_{\alpha}=h^{1 / 2}$.) Suppose $E^{*}$ is ordered arbitrarily. Let $K(A)$ be the matrix representation of $K(A)$ with respect to $E^{*}$. It is known that if $A \geqq 0$, so is $K(A)$, and $A \geqq B$ implies $K(A) \geqq K(B)$ (see [6]). 
Now one can easily verify that the main diagonal entry in $\boldsymbol{K}(\boldsymbol{A})$ corresponding to the sequence $(1, \cdots, n)$ is

$$
\begin{aligned}
& h\left(K(A) e_{1} * \cdots * e_{n}, e_{1} * \cdots * e_{n}\right) \\
& \quad=h\left(A e_{1} * \cdots * A e_{n}, e_{1} * \cdots * e_{n}\right)=d(A) .
\end{aligned}
$$

If $A \geqq B$ and $d(A)=d(B)$ then, being $\geqq 0, \boldsymbol{K}(\boldsymbol{A})-\boldsymbol{K}(\boldsymbol{B})$ has a zero row and column corresponding to $(1, \cdots, n)$. It follows that

$$
A e_{1} * \cdots * A e_{n}=B e_{1} * \cdots * B e_{n} .
$$

For $\chi \equiv 1$, we can apply Lemma 2.4 of [5] to (4) to see that $A=B Q$, where $Q$ is a monomial whose entry in column $j$ is $d_{j}$. Since $d(A)=d(B) \neq 0$, we have $\Pi_{j} d_{j}=1$. Since $B \geqq 0,\left|b_{i j}\right|^{2} \leqq b_{i i} b_{j j}$ for all pairs $i, j$. If $\sigma \in S_{n}$ is the permutation corresponding to $Q$, then $a_{j j}=b_{\sigma(j) j} d_{j} \leqq b_{\sigma(j) \sigma(j)}^{1 / 2} b_{j j}^{1 / 2}\left|d_{j}\right|$. Hence, $\prod_{j} a_{j j}=\prod_{j} b_{j j}$. But, $A \geqq B$ implies $a_{j j} \geqq b_{j j} \geqq 0$. Also, since $d(B) \neq 0$, no row of $B$ can be zero, and hence $b_{j j} \neq 0$ for all $j$. Therefore $a_{j j}=b_{j j}$, $1 \leqq j \leqq n$, or $\operatorname{trace}(A-B)=0$, and the result is established.

The authors thank the referee for shortening the proof of Theorem 1.

\section{REFERENCES}

1. E. F. Beckenbach and R. Bellman, Inequalities, Ergebnisse der Mathematik und ihrer Grenzgebiete, Heft 30, Springer-Verlag, Berlin, 1961. MR 28 \#1266.

2. R. Bellman, Notes on matrix theory. II, Amer. Math. Monthly 60 (1953), 173-175. MR 14, 731.

3. Ralph Freese, Inequalities for generalized matrix functions based on arbitrary characters, Linear Algebra and Appl. (to appear).

4. Marvin Marcus and Henryk Minc, A survey of matrix theory and matrix inequalities, Allyn and Bacon, Boston, Mass., 1964. MR 29 \#112.

5. ——, Generalized matrix functions, Trans. Amer. Math. Soc. 116 (1965), 316329. MR 33 \#2655.

6. Marvin Marcus and Paul J. Nikolai, Inequalities for some monotone matrix functions, Canad. J. Math. 21 (1969), 485-494. MR 38 \#5815.

7. L Mirsky, An inequality for positive definite matrices, Amer. Math. Monthly 62 (1955), 428-430. MR 17, 338.

8. I. Schur, Über endliche Gruppen und Hermitesche Formen, Math. Z. 1 (1918), 184-207.

National Bureau of Standards, Washington, D.C. 20234

Current address (Merris): Department of Mathematics, California State College, Hayward, California 94542

Current address (Pierce): Department of Mathematics, University of Toronto, Toronto 181, Ontario, Canada 